\title{
Nasca classification of hemivertebra in five dogs
}

Omer Besalti, Ahmet Ozak, Zeynep Pekcan and Salih Eminaga

\section{Keywords:}

Dog,

Vertebral anomaly,

Hemivertebra,

Five dogs, four small mixed breed and a Doberman Pinscher, presented in our clinic with hemivertebra. Nasca classification. Complete physical, radiological and neurological examinations were done and the spinal deformities were characterized in accord with the Nasca classification used in human medicine. Two dogs had multiple hemivertebrae (round, oval or wedge-shaped:Type 3) in the thoracic region; one dog had an individual surplus half vertebral body (Type I) plus a wedge-shaped hemivertebra (Type 2b) in the lumbar region; one dog had multiple hemivertebrae which were fused on one side (Type 4a) in the thoracic region; and one dog had a wedge-shaped hemivertebra (Type 2a) in the cervical region.

Irish Veterinary Journal

Volume 58: 688-690, 2005

\section{Introduction}

Congenital spinal deformities in companion animals are reported as hereditary disorders (Parker et al., 1973; Johnson et al., 1997; Morgan, 1999; Done et al., 1975; Nagahata et al., 2002). Because of the importance of the intersegmental artery in the formation of the definitive vertebral body anlage, it may be concluded that congenital vertebral malformations are likely to occur during the stage of segmentation and to be related to the abnormal distribution of the intersegmental arteries (Bailey and Morgan, 1992). Congenital spinal deformity manifests itself differently depending on localization and the involvement of neural structures; in general, it tends to be progressive in nature (Done et al., 1975).

In human medicine, the Nasca classification of spinal deformity is used for determining prognosis and treatment models of the condition (Nasca et al., 1975; Birnbaum et al., 2002). In this communication, we used the Nasca system to classify five cases of hemivertebra that were presented at our clinic.

\section{Case reports}

Between March 1998 and January 2003, five dogs with congenital spinal deformity were presented to the Department of Surgery, Faculty of Veterinary Medicine, Ankara University. Case details are recorded in Table I. Complete physical, radiological and neurological examinations were done. The Nasca classification (Nasca et al., 1975; Birnbaum et al., 2002) was adopted in describing each spinal deformity (Figure I).

\section{Correspondence:}

\section{Omer Besalti}

Department of Surgery, Faculty of Veterinary Medicine, Ankara University, Dıskapi, Ankara, 061 10, Turkey.

Tel: +90 (3।2) 317 03।5; Fax: +90 (3।2) 316 4472; E-mail: besalti@ hotmail.com.

\section{Clinical investigations}

Progressive hindlimb weakness, muscle atrophy and paraparesis or paraplegia were reported concurrently in dogs I, 2 and 4. In dogs I, 2, 3 kyphosis, lordosis and scoliosis at thoracic vertebra were apparent on physical examination. In dog 4 there was no apparent physical abnormality. Dogs I and 4 had hindlimb ataxia, conscious proprioceptive deficit and incoordination. Dog 2 had upper motor neuron deficits and inability to stand on its hindlimbs. Dogs 3 and 5 exhibited non-ambulatory tetraparesis.

The spinal deformity was seen in all dogs in plain lateral radiographs; however, superposition of structures make interpretation difficult in ventrodorsal radiographs. In each case, the disorder was classified according to the Nasca scheme. Two dogs had multiple hemivertebrae (round, oval or wedge-shaped:Type 3) in the thoracic region; one dog had an individual surplus half vertebral body (Type I) plus a wedgeshaped hemivertebra (Type 2b) in the lumbar region; one dog had multiple hemivertebrae which were fused on one side (Type 4a) in the thoracic region; and one dog had a wedge-shape hemivertebra (Type $2 a)$ in the cervical region.

Complete arrest of contrast flow was observed on the myelograms of the three dogs in which lordosis-kyphosis-scoliosis was observed on physical examination; in these dogs there were multiple anomalies (Figure 2). In dog 5, myelography revealed dorsal displacement of wedge-shaped hemivertebra, narrowing of the vertebral canal and spinal cord compression.

Four dogs with thoracic and lumbar hemivertebrae (Figure 3) were euthanized at the request of the owners. Dog 5 had undergone unsuccessful surgical intervention and was euthanized at the request of the owner because of neurological deterioration by the fifteenth day post-operation.

\section{Discussion}

Congenital spinal deformity has been reported in dogs, horses, and a calf (Johnson et al., 1997; Morgan, 1999; Done et al., 1975; Nagahata et al., 2002). Hemivertebra has been seen most commonly 


\begin{tabular}{lllllll} 
Case No & Breed & Age & $\begin{array}{l}\text { Gender } \\
\text { status }\end{array}$ & Neurological & Table I: Details of the five cases of hemivertebra in dogs \\
Involved & vertebrae & $\begin{array}{l}\text { Nasca } \\
\text { classification }\end{array}$ \\
\hline I & Mixed & $6 \mathrm{~m}$ & Male & Paraparesis & T5 - T9 & Multiple hemivertebra Type 3 \\
\hline 2 & Mixed & $3 \mathrm{~m}$ & Female & Paraplegia & T3 - T9 & Multiple hemivertebrae Type 3 \\
\hline 3 & Mixed & $3 \mathrm{~m}$ & Male & Nonambulatory tetraparesis & C7 - T7 & Multiple hemivertebra Type 4a \\
\hline 4 & Mixed & $4 \mathrm{~m}$ & Female & Paraparesis & L2 - L3 & L3 hemivertebra Type I \\
L2 hemivertebra Type 2b
\end{tabular}

in screw-tailed breeds (pugs, bulldogs and Boston terriers), in which it is responsible for the kink in the tail. In this small series the breed dispersion did not conform to that pattern, in that four of the five dogs were of mixed parentage, unrelated to screw-tailed breeds.

In humans, mutations in genes required for the intrinsic biochemical regulation of segmentation of the vertebral column have been implicated in spinal deformities (Pourquié and Kusumi, 200I). In addition, the roles of some environmental factors have been studied experimentally by medical authors (Loder et al., 2000; Debouck et al., 200I; Kaiser et al., 2003; Wéry et al., 2003). There was nothing in the history of the five dogs in the present series that pointed to particular genetic or environmental factors that might have caused the anomalies or predisposed the subjects to them.

In humans, the description of spinal deformity was classified by Nasca and his colleagues and that classification has been used for

Type I is characterized by an individual surplus half vertebral body. It is mostly round or oval and localized between two adjoining vertebral bodies. In the course of time, it often merges with one or both adjacent vertebral bodies. In the thoracal region, the surplus vertebral body is associated with an additional rib and regular vertebral arch oval.

Type 2 can be a wedge-shaped hemivertebra or a wedge-shaped vertebra. It mostly has a triangular configuration. It is not associated with an additional rib at the thoracic spine. Subtype $\mathbf{2 a}$ (wedgeshaped hemivertebra) represents a hemivertebra for which the ontogenesis of the other side of the vertebral body as well as of the neural tube has completely failed. Subtype $\mathbf{2 b}$ (designated by a wedge-shaped vertebra) is a hemivertebra in which one side of the vertebral body and of the neural tube is underdeveloped.

Type 3 is characterized by multiple hemivertebra that can be round, oval, or wedge-shaped.

Type 4 is defined by appearance of multiple hemivertebra that are fused on one side (so called unilateral bar). There is a further differentiation into two subtypes. Subtype $\mathbf{4 a}$ is characterized by multiple hemivertebrae with one-sided fusion of vertebral bodies and of the posterior elements of the vertebral body on the concave side. Subtype $\mathbf{4 b}$ is characterized by multiple wedge-shaped vertebrae with developing one-sided fused vertebrae.

Type 5 represents balanced hemivertebrae, localized in such a way that the deforming effects are neutralized by each other. In this way, an extreme scoliosis form can be avoided. determining prognosis and, also, treatment models of scoliosis (Nasca et al., 1975; Birnbaum et al., 2002). To the authors' knowledge, this is the first report to use the Nasca classification in a study of congenital spinal deformity in the dog.

Contrary to previous literature, which reported that only a single vertebra was involved in most cases (Done et al., 1975; Widmer, 1980), four of the five cases in this series had multiple spinal deformities: in the thoracic region in three dogs, in lumbar vertebrae in one dog.

Caudal cervical vertebral instability is well known in the dog (McKee and Sharp, 2003). The Doberman Pinscher (case 5) had a wedgeshaped cervical vertebra that induced instability and compression on the spinal cord. As far as we know, this is the first report implicating cervical hemivertebra as a cause of caudal cervical vertebral instability in the dog.

Type $\mathbf{6}$ is defined by posterior hemivertebrae that lead to a progressive kyphosis rather than a scoliosis. A kyphosis arises when the anterior part of the vertebral body does not develop as an independent unit.

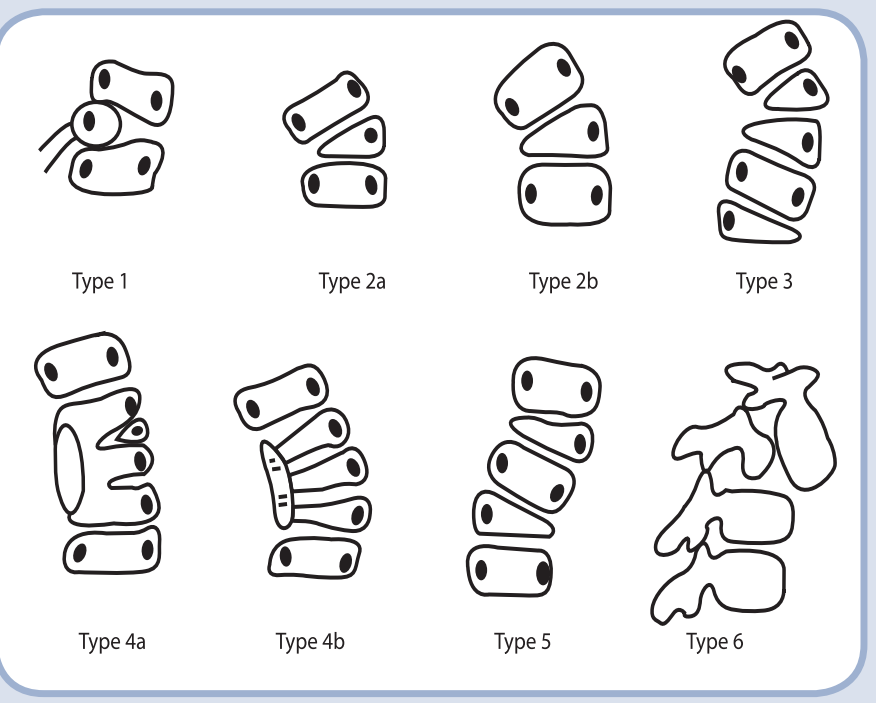

Figure I: Drawings of the six types of vertebral anomaly as defined by Nasca et al. (1975), with explanation of the different types (Birnbaum et al., 2002). Figure I has been reproduced with kind permission of Springer Science and Business Media. 


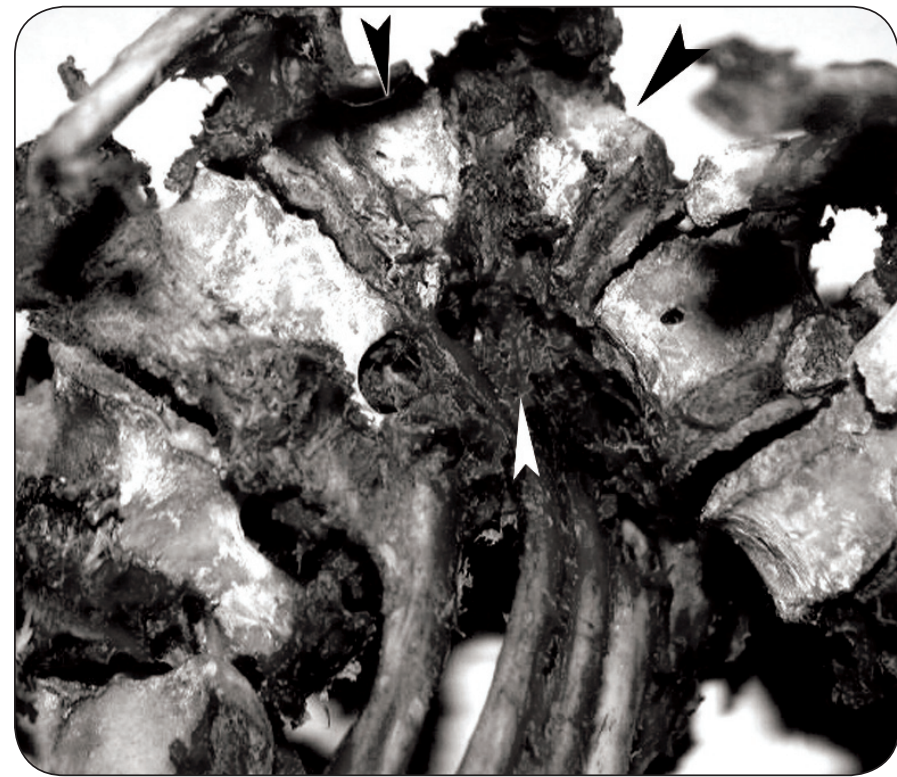

Figure 2:The photographic view of the multiple hemivertebrae (white arrow head) with one-sided fused vertebral bodies (black arrow head) in Case 3.

\section{References}

Bailey, C. S. and Morgan, J. P. (1992). Congenital spinal malformations. Veterinary Clinics of North America: Small Animal Practice 22: $985-1016$.

Birnbaum, K., Weber, M., Lorani, A., Leiser-Neef, U. and Niethard, F.U. (2002). Prognostic significance of the Nasca classification for the long-term course of congenital scoliosis. Archives of Orthopaedic and Trauma Surgery I 22: 383-389.

Debouck, C., Haubruge, E., Bollaerts, P., van Bignoot, D., Brostaux, Y., Werry, A. and Rooze, M. (200I). Skeletal deformities induced by the intraperitoneal administration of deoxynivalenol (vomitoxin) in mice. International Orthopaedics 25: 194-198.

Done, S.H., Drew, R.A., Robins, G.M. and Lane, J.G. (1975). Hemivertebra in the dog: clinical and pathological observations. Veterinary Record 96: 313-317.

Johnson, P. J., Johnson, G.C. and Pace, L.W. (1997). Thoracic vertebral malformation in two horses. Equine Veterinary Journal 29 493-496.

Kaiser, M.E., Merrill, R.A., Stein, A.C., Breburda, E. and Clagett-Dame, M. (2003). Vitamin A deficiency in the late gastrula stage rat embryo results in a one to two vertebral anteriorization that extends throughout the axial skeleton. Developmental Biology 257 14-29.

Loder, R.T., Hernandez, M.J., Lemer, A.L., Winebrener, D.J., Goldstein, S.A., Hensinger, R.N., Liu, C.-Y. and Schork, M.A. (2000). The induction of congenital spinal deformities in mice by

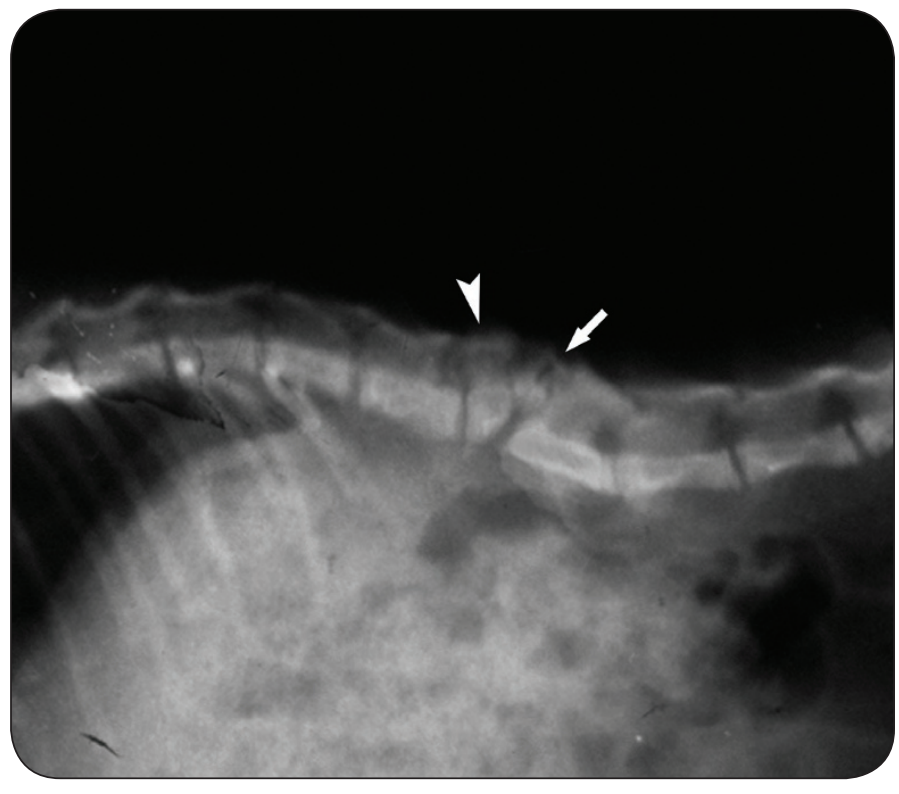

Figure 3:The radiographic view of Type I (L3, arrow) and Type 2 (L2, arrow head) hemivertebrae according to the Nasca classification (Case 4).

maternal carbon monoxide exposure. Journal of Pediatric Orthopaedics 20: 662-666.

McKee, W.M. and Sharp, N. (2003). Cervical spondylopathy. In: Textbook of Small Animal Surgery, Third edition, Volume I, pp I I80-1।93, Edited by D. Slatter. Philadelphia: Saunders.

Morgan, J.P. (1999). Transitional lumbosacral vertebral anomaly in the dog: a radiographic study. Journal of Small Animal Practice 40: I67- 172.

Nagahata, H., Oota, H., Nitanai, A., Oikawa, S., Higuchi, H., Nakade, T., Kurosawa, T., Morita, M. and Ogawa, H. (2002). Complex vertebral malformation in a stillborn Holstein calf in Japan. Journal of Veterinary Medical Science 64: I I 07- I I I 2.

Nasca, R.J., Stelling, F.H. and Steel, H.H. (1975). Progression of congenital scoliosis due to hemivertebrae and hemivertebrae with bars. Journal of Bone and Joint Surgery American Edition 57: 456-466.

Parker, A.J., Park, R.D. and Stowater, J.L. (1973). Cervical kyphosis in an Afghan Hound. Journal of the American Veterinary Medical Association 162: 953-955.

Pourquie, O. and Kusumi, K. (200I). When body segmentation goes wrong. Clinical Genetics 60: 409-416.

Wery, N., Narotsky, M.G., Pacino, N., Kavlock, R.J., Picard, J. J. and Gofflot, F. (2003). Defects in cervical vertebrae in boric acidexposed rat embryos are associated with anterior shifts of hox gene expression domains. Birth Defects Research (Part A) 67: 59-67.

Widmer W.R. (1980). What is your diagnosis? Journal of the American Veterinary Medical Association 176: I0I7-1018. 